\title{
REGULARITY OF THE METRIC ENTROPY FOR EXPANDING MAPS
}

\author{
MAREK RYCHLIK
}

\begin{abstract}
The main result of the current paper is an estimate of the radius of the nonperipheral part of the spectrum of the Perron-Frobenius operator for expanding mappings. As a consequence, we are able to show that the metric entropy of an expanding map has modulus of continuity $x \log (1 / x)$ on the space of $C^{2}$-expandings. We also give an explicit estimate of the rate of mixing for $C^{1}$-functions in terms of natural constants.

It seems that the method we present can be generalized to other classes of dynamical systems, which have a distinguished invariant measure, like Axiom A diffeomorphisms. It also can be adopted to show that the entropy of the quadratic family $f_{\mu}(x)=1-\mu x^{2}$ computed with respect to the absolutely continuous invariant measure found in Jakobson's Theorem varies continuously (the last result is going to appear somewhere else).
\end{abstract}

\section{INTRODUCTION}

1.1 Formulating the problem. In many situations one would like to consider families of dynamical systems rather than individual mappings or flows. In these situations a natural question arises of how numerical invariants of the dynamical system, such as the topological or metric entropy, depend on the parameters. The continuity of the topological entropy has been studied extensively (for further references on the subject $\mathrm{cf}$. [12]). In the current paper we address the problem of the regularity of the metric entropy for systems which have a naturally distinguished invariant measure. We concentrate on the best understood class of such systems, the class of expanding mappings. It is well known (cf. [5]) that these mappings have a unique probabilistic invariant measure absolutely continuous with respect to the Lebesgue measure.

We do not attempt to carry out a similar analysis for more complicated dynamical systems here. However, there are other situations where similar results have been obtained. This includes Axiom A systems, for which the natural class of invariant measures is the class of Sinai-Bowen-Ruelle measures.

Received by the editors April 28, 1988.

1980 Mathematics Subject Classification (1985 Revision). Primary 58F11.

This paper was written during the author's stay at the IAS while supported by NSF grant DMS8601978. 
The problem for expanding mappings has an elegant solution, which should be treated as a model for other classes of systems. The most complicated situation to which this model can be applied is the quadratic family $f_{\mu}(x)=1-\mu x^{2}$. By the theorem of Jakobson [4] there is a set of parameters $\mu$ of positive measure for which the mapping $f_{\mu}$ has an absolutely continuous invariant measure. For the set of parameters found in [8] the measure is unique. By a generalization of the methods of this paper we are able to show that the Lyapunov exponent of the mapping $f_{\mu}$ varies continuously on that set. The intermediate result is that the density of the invariant measure depends continuously on the parameter in $L^{1}$-topology. These results will be presented in [9].

1.2 A brief statement of the main result. For a $C^{2}$-expanding map $\varphi: M \rightarrow M$, Krzyżewski and Szlenk [5] showed the existence of a function $h_{\varphi}: M \rightarrow \mathbf{R}_{+}$ of class $C^{1}$ such that the measure $\mu_{\varphi}=h_{\varphi} m$ is $\varphi$-invariant ( $m$ denotes the Riemann-Lebesgue measure corresponding to a fixed Riemannian metric). The main estimate of this paper implies that the map $\varphi \mapsto h_{\varphi}$ is continuous from the space of $C^{2}$-expanding maps with $C^{2}$-topology to $C^{0}(M, \mathbf{R})$ and the modulus of continuity is $x \log (1 / x)$. It is also true that this map is continuous from the space of $C^{2}$-expanding maps with $C^{2}$-topology to $C^{1}(M, \mathbf{R})$ (we will show that $\left.h_{\varphi} \in C^{1}(M, \mathbf{R})\right)$. However, the modulus of continuity requires a more complicated description, as it will be clear from the proof of Theorem 2 . We will also show the exponential mixing rate of $\varphi$ for $C^{2}$-functions with a direct estimate. The new result in the context of expanding maps is that the rate of mixing can be estimated in terms of the expansion constant, the distorsion and the diameter of the manifold. Precise statements will be given later. Hence, the mixing estimate is uniform on any compact family of $\varphi$ 's.

1.3 The method. The problem formulated in $\S 1.2$ translates into a perturbation problem for the Perron-Frobenius operator (abbreviation: P-F) of an expanding map. Subsequently, an estimate of the nonperipheral part of the spectrum of the P-F (Perron-Frobenius) operator (Theorem 1) is given. It is well known that such estimates yield the rate of mixing for certain classes of functions [2, 3]. We will show an explicit bound for the rate of mixing for $C^{1}$-functions in terms of some naturally defined constants.

1.4 The rate of mixing. In the next few paragraphs we will describe the connection between the rate of mixing of an expanding map $\phi$ and bounds for the nonperipheral part of the spectrum of the P-F operator.

Let $f, g \in C^{1}(M, \mathbf{R})$. We consider the sequence $\left(\mu=\mu_{\varphi}\right)$

$$
k_{n}=\int_{M} f \circ \varphi^{n} \cdot g d \mu-\int_{M} f d \mu \int_{M} g d \mu .
$$

For expanding mappings the existence of Markov partitions [6] combined with the paper of Bowen [2] yields an estimate $\left|k_{n}\right| \leq$ const $\cdot r^{n}$ where $r \in(0,1)$ is 
independent of $f$ and $g$. The P-F operator $P_{\varphi}: L^{1}(m) \rightarrow L^{1}(m)$ is uniquely determined by the property: for every $f \in L^{\infty}(m), g \in L^{1}(m)$ :

$$
\int_{M} f \circ \varphi^{n} g d m=\int_{M} f P_{\varphi}^{n} g d m
$$

(In other words; $P_{\varphi}^{*}=U_{\varphi}$, where $U_{\varphi}(f)=f \circ \varphi$.) Using this identity, we can write

$$
k_{n}=\int_{M} f\left(P^{n}\left(g \cdot h_{\varphi}\right)-\int_{M}\left(g \cdot h_{\varphi}\right) d m h_{\varphi}\right) d m .
$$

Let us introduce a projection $Q_{\varphi}: L^{1}(m) \rightarrow L^{1}(M)$ via the formula

$$
Q_{\varphi}(g)=\int_{M} g d m \cdot h_{\varphi} .
$$

One can show that $P_{\varphi}$ is bounded as an operator from $C^{1}$ to $C^{1}$. It proves that the spectrum of the P-F operator $P_{\varphi}$ consists of 1 and a set contained within a disk of radius $r_{0}<1$ called a nonperipheral spectrum of $P_{\varphi}$ (this result is true in view of [2]; a more up to date way of proving such estimates is in $[3,7])$.

From the definition of $r_{0}$ it follows that for any $r>r_{0}$ :

$$
\left\|P_{\varphi}^{n}-Q_{\varphi}\right\|_{C^{1}} \leq \text { const } \cdot r^{n} \text {. }
$$

We can apply this inequality and (3) for any function $\chi \in C^{1}(M, \mathbf{R})$ to obtain

$$
\left|k_{n}\right| \leq \text { const }\|f\|_{L^{1}}\|\chi\|_{C^{1}} r^{n} \text {. }
$$

On the other hand, if $r<r_{0}$ then one can pick functions $f$ and $\chi$ in such fashion that $\left|k_{n}\right| r^{-n} \rightarrow \infty$. So, roughly speaking, $r_{0}$ is the rate of mixing for $C^{1}$-functions.

1.5 Continuity of the metric entropy. Another application of our estimate of the nonperipheral part of the spectrum of the P-F operator is a proof of the continuity of $h_{\varphi}$ as a function of $\varphi$. One can develop the right approach as follows: suppose that $\varphi, \psi$ are two close expanding mappings and let us write $f=h_{\varphi}-h_{\psi}$. Then one has

$$
P_{\varphi}\left(h_{\psi}+f\right)=h_{\psi}+f
$$

or

$$
\left(I-P_{\varphi}\right) f=P_{\varphi} h_{\psi}-h_{\psi} \stackrel{\text { def }}{=} u .
$$

In general this equation is not solvable, since $1 \in \operatorname{spec}\left(P_{\varphi}\right)$. We notice, though, that $m(u)=m\left(P_{\varphi} h_{\psi}\right)-m\left(h_{\psi}\right)=m\left(h_{\psi}\right)-m\left(h_{\psi}\right)=0$. (Notice that $m(P f)=$ $m(f)$ for any $f \in L^{1}(m)$.) The spectral radius of the operator $P_{\varphi}$ restricted 
to $X=\left\{f \in C^{1}: m(f)=0\right\}$ is $r_{0}<1$. Hence the solution to (8) can be given by a convergent von Neumann series:

$$
f=\left(I-P_{\varphi}\right)^{-1} u=\sum_{n=0}^{\infty} P_{\varphi}^{n} u .
$$

A more precise analysis will show that if $\psi$ is $C^{2}$-close to $\varphi$ then $u$ is small in $C^{0}$-topology and uniformly bounded in $C^{1}$-topology. This is sufficient to show that $f$ is small in $C^{0}$-topology.

We recall that for expanding mappings we have the following version of Rokhlin-Pěsin's formula [11]:

$$
h_{\mu_{\varphi}}(\varphi)=\int_{M} \log J_{\varphi} d \mu_{\varphi}=\int_{M}\left(\log J_{\varphi}\right) \cdot h_{\varphi} d m .
$$

Since obviously $C^{2} \in \varphi \mapsto J_{\varphi} \in C^{1}$ is continuous, we obtain that the entropy $h_{\mu_{\varphi}}(\varphi)$ is a continuous function of $\varphi$ in $C^{2}$-topology. We will actually show that the modulus of continuity is of type $x \log (1 / x)$.

1.6 A remark on the analytic families. It is not difficult to show that for realanalytic families of expanding mappings the density of the absolutely continuous invariant measure is an analytic function of the parameter. It is due to the compactness of the Perron-Frobenius operator in the analytic domain. However, even in this situation our bound for the rate of mixing seems to be the only explicit bound known.

1.7 Acknowledgements. The author would like to acknowledge kindly support of the Institute for Advanced Study during writing this paper. The author expresses his thanks to T. Spencer, whose suggestions led to formulating the problem, and to E. Sorets and M. Pollicott for helpful conversations during the writing of this paper.

\section{Preliminary Considerations}

The content of this section is introductory material concerning expanding mappings. Similar results can be found in the literature. We included full proofs of the facts we need for the convenience of the reader.

2.1 Basic notation. Let $\varphi: M \rightarrow M$ be a $C^{2}$-expanding map of a Riemannian manifold $M$. By $T_{x} M$ we denote the tangent space of $M$ at $x$. By $T_{x} \varphi: T_{x} M \rightarrow T_{\varphi(x)} M$ we denote the tangent map of $\varphi$ at $x \in M$. The norm induced by the Riemannian metric will be denoted by $|\cdot|$. Thus there are constants $C_{0} \in \mathbf{R}_{+}$and $\lambda \in(0,1)$ such that for every $x \in M$ :

$$
\left|\left(T_{x} \varphi^{n}\right)^{-1}\right| \leq C_{0} \lambda^{n}
$$

By $J$ we denote the Jacobian of $\varphi$, i.e.:

$$
J(x)=\left|\operatorname{det}\left(T_{x} \varphi\right)\right| .
$$


The determinant is computed with respect to any pair of orthonormal bases. The inverse of the Jacobian will be denoted by $g$, i.e.

$$
g(x)=1 / J(x) \text {. }
$$

We also introduce the analogous functions for the iterations:

$$
\begin{aligned}
& J_{n}(x)=J(x) \cdot J(\varphi x) \cdots J\left(\varphi^{n-1} x\right) \\
& g_{n}(x)=g(x) \cdot g(\varphi x) \cdots \cdot g\left(\varphi^{n-1} x\right) .
\end{aligned}
$$

Let $u=\log J$. From our assumptions it follows that $u$ is $C^{1}$ and

$$
C_{1}=\sup _{x \in M}|d u(x)|=\sup _{x \in M} \frac{|d J(x)|}{J(x)}
$$

is finite.

2.2 Basic properties of expanding maps. From the fact that $\varphi^{n}$ is a covering map several important facts follow. Let $x, y \in M$ and let $\sigma:[a, b] \rightarrow M$ be any $C^{1}$-path connecting $x$ to $y$. For any $n$ and any $x^{\prime} \in \varphi^{-n}(y)$ one can find a lifting path $\sigma_{n}:[a, b] \rightarrow M$ connecting $x^{\prime}$ to some $y^{\prime} \in \varphi^{-n}(y)$. We will refer to this construction by saying that the preimages $x^{\prime}$ and $y^{\prime}$ correspond by $\sigma$. It is easy to see that $l\left(\sigma_{n}\right) \leq l(\sigma) \cdot C_{0} \lambda^{n}$. By picking the path $\sigma$ to be the shortest geodesic path we show easily:

Lemma 1. For any $\varepsilon>0$ and any $n \in \mathbf{Z}_{+}$such that $\varepsilon>C_{0} \lambda^{n} \operatorname{diam}(M)$ and any $x \in M$ the set $\varphi^{-n}(x)$ is $\varepsilon$-dense in $M$.

Definition 1. A continuous map $\psi: X \rightarrow X$, where $X$ is a topological space, is called topologically mixing if for all nonempty open sets $U, V \subset X$ there is $n_{0} \in \mathbf{Z}_{+}$such that for every $n \geq n_{0}$ we have $\psi^{n}(U) \cap V \neq 0$.

Lemma 1 implies immediately that any expanding map is topologically mixing. The next lemma is a version of the well-known distorsion lemma.

Lemma 2. Suppose that $x, y \in M, \sigma:[a, b] \rightarrow M$ is $C^{1}$ and $x^{\prime} \in \varphi^{-n}(x)$, $y^{\prime} \in \varphi^{-n}(y)$ correspond to $\sigma$. Then

$$
\exp \left(-C_{2} l(\sigma)\right) \leq \frac{g_{l}\left(x^{\prime}\right)}{g_{l}\left(y^{\prime}\right)} \leq \exp \left(C_{2} l(\sigma)\right)
$$

where $C_{2}=C_{0} C_{1} \lambda /(1-\lambda)$.

Proof. Let $\sigma_{i}$ denote the lifting of $\sigma$ by means of $\varphi^{i}$ which connects $\varphi^{n-i}\left(x^{\prime}\right)$ to $\varphi^{n-i}\left(y^{\prime}\right)$. Clearly, we have

$$
l\left(\sigma_{i}\right) \leq C_{0} \lambda^{i} l(\sigma)
$$

As a result, we can write

$$
\begin{aligned}
& \left|\sum_{i=1}^{n}\left(u\left(\varphi^{n-i}\left(x^{\prime}\right)\right)-u\left(\varphi^{n-i}\left(y^{\prime}\right)\right)\right)\right| \\
& \quad \leq \sum_{i=1}^{n} C_{1} l\left(\sigma_{i}\right) \leq \sum_{i=1}^{n} C_{1} C_{0} \lambda^{i} l(\sigma) \leq C_{2} l(\sigma) .
\end{aligned}
$$


Obviously $\log g_{l}=-\sum_{i=0}^{l-1} u \circ \varphi^{i}$. Hence (18) implies that

$$
\left|\log g_{l}\left(x^{\prime}\right)-\log g_{l}\left(y^{\prime}\right)\right| \leq C_{2} l(\sigma) .
$$

This, in turn, implies (16).

\subsection{The definition and some properties of the P-F operator.}

Definition 2. The P-F operator of $\varphi$ is the following operator $P: C^{0}(M) \rightarrow$ $C^{0}(M)$ :

$$
P f(x)=\sum_{y \in \varphi^{-1}(x)} f(y) g(y) .
$$

It is easy to verify that

1. $m(P f)=f$, where $m$ is the Lebesgue measure on $M$;

2. $f \geq 0 \Rightarrow P f \geq 0$;

3. for every $n \in \mathbf{Z}_{+}$

$$
P^{n} f(x)=\sum_{y \in \varphi^{-n}(x)} f(y) g_{n}(y) ;
$$

4. $\left(\varphi^{n}\right)_{*}(f \cdot m)=\left(P^{n} f\right) \cdot m$ for every $f \in C^{0}(M)$ and $n \in \mathbf{Z}_{+}$.

One can use the next lemma to give a proof of Theorem 7.9 of Krzyżewski and Szlenk [5].

Lemma 3. Let us define

$$
S(\rho)=\left\{f \in C^{0}(M): f(x)>0 \text { for all } x \in M \text { and }(*)\right\}
$$

where (*) for every $x, y \in M$

$$
\exp (-\rho d(x, y)) \leq f(x) / f(y) \leq \exp (\rho d(x, y)) .
$$

Then for any integer $n \geq 1$ we have $P^{n}(S(\rho)) \subseteq S\left(\rho^{\prime}\right)$, where

$$
\rho^{\prime}=\rho C_{0} \lambda^{n}+C_{2} \text {. }
$$

Proof. One sees easily that if $f \in S(\rho)$ then

$$
\frac{P^{n} f(x)}{P^{n} f(y)} \leq \max _{\left(x^{\prime}, y^{\prime}\right)} \frac{f\left(x^{\prime}\right) g_{n}\left(x^{\prime}\right)}{f\left(y^{\prime}\right) g_{n}\left(y^{\prime}\right)},
$$

where the maximum is over all pairs $x^{\prime} \in \varphi^{-n}(x), y^{\prime} \in \varphi^{-n}(y)$ which correspond by some geodesic path $\sigma$ on $M$ connecting $x$ to $y$ of length $d(x, y)$. Therefore,

$$
\begin{aligned}
\frac{P^{n} f(x)}{P^{n} f(y)} & \leq \exp \left(\rho C_{0} \lambda^{n} d(x, y)\right) \exp \left(C_{2} d(x, y)\right) \\
& =\exp \left(\left(\rho C_{0} \lambda^{n}+C_{2}\right) d(x, y)\right) \\
& =\exp \left(\rho^{\prime} d(x, y)\right)
\end{aligned}
$$

The theorem has been proved. 
Corollary 1. For $n$ sufficiently large we have $C_{0} \lambda^{n}<1$. We define $\rho=$ $C_{2} /\left(1-C_{0} \lambda^{n}\right)$. For some $h \in S(\rho)$ we have $P^{n} h=h$, by the Schauder-Tychonoff fixed point theorem. The last lemma implies that for any $x, x^{\prime} \in M$

$$
\begin{aligned}
\exp \left(C_{2}^{-1} d\left(x, x^{\prime}\right)\right) & \leq h(x) / h\left(x^{\prime}\right) \leq \exp \left(C_{2} d\left(x, x^{\prime}\right)\right), \\
C_{3}^{-1} & \leq h(x) \leq C_{3}
\end{aligned}
$$

where $C_{3}=\exp \left(C_{2} \operatorname{diam}(M)\right)$.

We will now derive an inequality which has been most important in the study of the P-F operator.

Lemma 4. For every $f \in C^{1}(M)$ and $l \in \mathbf{Z}_{+}$we have

$$
\left|d\left(P^{l} f\right)\right| \leq C_{0} \lambda^{l} P(|d f|)+C_{2} P^{l}(|f|) .
$$

Proof. Locally we can represent the P-F operator of $\varphi$ as follows:

$$
P^{l} f(x)=\sum_{i=1}^{N(l)}\left(g_{l} \cdot f\right) \circ \phi_{l, i}(x)
$$

where $\phi_{l, i}$ are local right inverses of $\varphi^{l}$ and $N(l)=\operatorname{deg}(\varphi)^{l}$. Hence,

$$
\begin{aligned}
\left|d\left(P^{l} f\right)\right|= & \left|\sum_{i=1}^{N(l)}\left(d g_{l} \cdot f+g_{l} \cdot d f\right) \circ \phi_{l, i} \cdot d \phi_{l, i}\right| \\
\leq & C_{0} \lambda^{l} \sum_{i=1}^{N(l)}\left(g_{l} \cdot|d f|\right) \circ \phi_{l, i} \\
& +\max _{1 \leq i \leq N(l)} \frac{\left|d\left(g_{l} \circ \phi_{l, i}\right)\right|}{g_{l} \circ \phi_{l, i}} \cdot P(|f|) .
\end{aligned}
$$

We also have

$$
\begin{aligned}
\left|\frac{d\left(g_{l} \circ \phi_{l, i}\right)}{g_{l} \circ \phi_{l, i}}\right| & =\left|\sum_{j=0}^{l-1} d\left(u \circ \varphi^{j} \circ \phi_{l, i}\right)\right| \\
& \leq \sum_{j=0}^{l-1}|d u| \circ\left(\varphi^{j} \circ \phi_{l, i}\right) \cdot\left|D\left(\varphi^{j} \circ \phi_{l, i}\right)\right| \\
& \leq \sum_{j=0}^{l-1} C_{1} C_{0} \lambda^{n-j} \leq C_{1} C_{0} \lambda /(1-\lambda)=C_{2} .
\end{aligned}
$$

This concludes our proof.

We shall define norms

$$
\begin{array}{r}
\|f\|=\sup _{M}|f| / h, \\
\|d f\|=\sup _{M}|d f| / h,
\end{array}
$$


where $h$ is a fixed function $h \in S$ such that $P h=h$ (it is well known that $h$ is unique up to a multiplicative constant, but this fact will be proved later independently). From now on we also assume that $h$ has been normalized, so that $\int_{M} h d m=1$. It is easy to verify that $\left\|P^{l} f\right\| \leq\|f\|$ for any $f \in C^{0}(M)$ and $l \in \mathbf{Z}_{+}$. This implies easily

Proposition 1. For any $f \in C^{1}(M, \mathbf{R})$ and $l \in \mathbf{Z}_{+}$

$$
\begin{aligned}
\left\|P^{l} f\right\| & \leq\|f\|, \\
\left\|d\left(P^{l} f\right)\right\| & \leq A \lambda^{l}\|d f\|+B\|f\|
\end{aligned}
$$

where $A=C_{0}$ and $B=C_{2}$.

Proof. We use the identity $f_{1} \cdot P f_{2}=P\left(f_{1} \circ T \cdot f_{2}\right)$. Hence

$$
\begin{aligned}
\frac{\left|d\left(P^{l} f\right)\right|}{h} & \leq C_{0} \lambda^{l} P\left(\frac{h}{h \circ T} \cdot \frac{|d f|}{h}\right)+C_{2} P\left(\frac{h}{h \circ T} \cdot \frac{|f|}{h}\right) \\
& \leq C_{0} \lambda^{l}\|d f\|+C_{2}\|f\| .
\end{aligned}
$$

Our proof has been completed.

Remark 1. One often defines the operator

$$
L f(x)=\sum_{y \in \varphi^{-1}(y)} p(y) f(y)=\frac{1}{h} P(f \cdot h)
$$

where $p=g \cdot h / h \circ T$. From the last proof it follows that $L$ is a (weak) contraction on $C^{0}(M)$.

Remark 2. It is known that $h \in C^{1}$ [6].

Let us discuss a property of expanding mappings which follows from the definition of cut locus and its basis properties.

Suppose that $x \in M$ and $\xi \in T_{x} M,|\xi|=1$. Let $a(\xi)$ be the smallest number in the set of $a>0$ such that the geodesic $t \mapsto \exp _{x}(t \xi), 0 \leq t \leq a$, does not minimize distance. One can show that the function $\xi \mapsto a(\xi)$ is continuous and the set $\widetilde{C}(x)=\{a(\xi) \xi:|\xi|=1\} \subset T_{x} M$ is called the cut locus of $x$. We observe that $\widetilde{C}(x)$ is homeomorphic to a sphere $|\xi|=1$. Let us consider the set $\widetilde{B}(x)=\{t \xi: 0 \leq t \leq a(\xi),|\xi|=1\}$. Clearly $\operatorname{int}(\widetilde{B}(x))=\{t \xi: 0 \leq$ $t \leq a(\xi),|\xi|=1\}$ is a topological ball. One shows that $\exp _{x}(\widetilde{B}(x))=M$ and clearly $m\left(\exp _{x}(\widetilde{C}(x))\right)=0$. Therefore one can think of $M$ as $\widetilde{B}(x)$ with some identifications on the boundary. Suppose $l \in \mathbf{Z}_{+}$and $x^{\prime} \in \varphi^{-l}(x)$. One can lift the map $\exp _{x}: \widetilde{B}(x) \rightarrow M$ via $\varphi^{l}$ and obtain a map $\exp _{x, x^{\prime}}: \widetilde{B}(x) \rightarrow M$. The sets $B_{l}\left(x^{\prime}\right)=\exp _{x, x^{\prime}}(\operatorname{int}(\widetilde{B}(x))$ have the following properties:

1. $m\left(M \backslash \bigcup_{x^{\prime} \in \varphi^{-1}(x)} B_{l}\left(x^{\prime}\right)\right)=0$;

2. $B_{l}\left(x^{\prime}\right) \cap B_{l}\left(x^{\prime \prime}\right)=0$, if $x^{\prime}, x^{\prime \prime} \in \varphi^{-l}(x), x^{\prime} \neq x^{\prime \prime}$;

3. $\operatorname{dist}\left(\left\{x^{\prime}\right\}, B_{l}\left(x^{\prime}\right)\right) \leq C_{0} \lambda^{l} \operatorname{diam}(M)$; 
4. $\operatorname{diam}\left(B_{l}\left(x^{\prime}\right)\right) \leq 2 C_{0} \lambda^{l} \operatorname{diam}(M)$;

5. $m\left(M \backslash \varphi^{l}\left(B_{l}\left(x^{\prime}\right)\right)\right)=0$.

\section{THE SPECTRUM OF THE P-F OPERATOR}

Throughout this section we will often write $\Delta$ instead of $\operatorname{diam}(M)$.

3.1 The main estimate (Theorem 1). Let $\varphi$ be a fixed piecewise expanding and let $P=P_{\varphi}$ be its P-F operator. The objective of this section is the following theorem.

Theorem 1. There is $l \in \mathbf{Z}_{+}$and constants $R \in \mathbf{R}, \gamma \in(0,1)$, which can be written in terms of $C_{0}, C_{1}, \lambda, \operatorname{diam}(M)$ only, such that $P^{l}$ is a $\gamma$-contraction of the Banach space $(X,|||\cdot|||)$ where

$$
X=\left\{f \in C^{1}: m(f)=0\right\}
$$

and $|\|\cdot \mid\|$ is defined as follows

$$
|\|f \mid\|=\max (\|d f\|, R\|f\|) .
$$

Proof. To show that $P^{l}$ is a contraction, we will pick a constant $\varepsilon>0, \gamma \in$ $(0,1)$ and consider two cases:

Case 1. $\|f\| \leq \varepsilon\|d f\|$,

Case 2. $\|f\| \geq \varepsilon\|d f\|$.

If $\varepsilon$ and $\gamma$ are chosen properly, we will show that in both cases ||$|\cdot| \mid$ gets contracted by $P^{l}$. The estimates are as follows:

Case 1 (easy). We have

$$
\begin{aligned}
R\left\|P^{l} f\right\| & \leq R\|f\| \leq R \varepsilon\|d f\| \leq R \varepsilon \mid\|f\| \\
\left\|d\left(P^{l} f\right)\right\| & \leq A \lambda^{l}\|d f\|+B\|f\| \leq\left(A \lambda^{l}+B \varepsilon\right)\|d f\| .
\end{aligned}
$$

As a result we obtain

$$
\left\|P ^ { l } f \left|\left\|\leq \max \left(R \varepsilon, A \lambda^{l}+B \varepsilon\right) \cdot|\|f \mid\| .\right.\right.\right.
$$

We will require that $\varepsilon, R, l$ are such that

$$
\max \left(R \varepsilon, A \lambda^{l}+B \varepsilon\right)<1 .
$$

Case 2 (the main estimate). We may assume that $\|f\|=1$ and $\|d f\| \leq \varepsilon^{-1}$. We further assume that $\left\|P^{l} f\right\| \geq \gamma\|f\|$ and we will show that for $\gamma$ near 1 we get a contradiction. Thus for some $x \in M\left(\hat{f} \stackrel{\text { def }}{=} f / h\right.$ and $\left.p_{l} \stackrel{\text { def }}{=} g_{l} \cdot h / h \circ T^{l}\right)$,

$$
\frac{P^{l} f(x)}{h(x)}=\sum_{y \in \varphi^{-l}(x)} p_{l}(y) \hat{f}(y) \geq \gamma \text {. }
$$


Let $Q=\left\{y \in \varphi^{-l}(x): \hat{f}(y) \geq \gamma / 2\right\}$. We have

$$
\sum_{y \in Q} p_{l}(y) \cdot 1+\sum_{y \in \varphi^{-l}(x) \backslash Q} p_{l}(y) \cdot \frac{\gamma}{2} \geq \gamma
$$

which leads to the estimate

$$
\sum_{y \in Q} p_{l}(y) \geq \frac{\gamma}{2}
$$

Let $\delta=\varepsilon \gamma / 8$. We claim that for $y \in Q$ we have $B(y, \delta) \subset\left\{y^{\prime} \in M: \hat{f}(y) \geq\right.$ $\gamma / 4\}$. Indeed, if it were not the case then there would be a point $y^{\prime} \in B(y, \delta)$ such that $\hat{f}\left(y^{\prime}\right)=\gamma / 4$ and $\hat{f}\left(y^{\prime \prime}\right) \geq \gamma / 4$ for any $y^{\prime \prime}$ on the geodesic path connecting $y$ to $y^{\prime}$. Along that path we can write the following inequalities:

$$
\|d f\| \leq \varepsilon^{-1} h ; \quad f \geq \frac{\gamma}{4} h .
$$

So

$$
\frac{d f}{f}=d(\log f) \leq \frac{\varepsilon^{-1}}{\gamma / 4}=\frac{4}{\varepsilon \gamma}
$$

Hence,

$$
\frac{f\left(y^{\prime}\right)}{f(y)} \geq \exp \left(-\frac{4}{\varepsilon \gamma} d\left(y^{\prime}, y\right)\right) \geq \exp \left(-\frac{4}{\varepsilon \gamma} \delta\right) \geq \frac{1}{\sqrt{e}} \geq \frac{1}{2}
$$

The claim has been proved.

Let us assume that $l$ is large enough, so that $\delta \geq \Delta \lambda^{l}$. This implies that $B_{l}(y) \subset B(y, \delta)$. Let $f_{+}$denote $\max (f, 0)$ for any function $f$ and let $\mu=h m$ be the invariant measure for $\varphi$. As a consequence we obtain

$$
\int f_{+} d m=\int \hat{f} d \mu \geq \sum_{y \in Q} \frac{\gamma}{4} \mu\left(B_{l}(y)\right) .
$$

The measure $\mu\left(B_{l}(y)\right)$ can be estimated as follows. We know that $\varphi^{l}\left(b_{l}(y)\right)$ $=M(\bmod 0)$. Therefore we can write

$$
\frac{\mu\left(B_{l}(y)\right)}{1}=\frac{\mu\left(B_{l}(y)\right)}{\mu\left(\varphi^{l}\left(B_{l}(y)\right)\right)} \geq \inf _{B_{l}(y)} p_{l}
$$

We also have: $p_{l}=g_{l} \cdot h / h \circ \varphi^{l}$ and for $y^{\prime} \in B_{l}(y)$

$$
\begin{aligned}
\frac{p_{l}\left(y^{\prime}\right)}{p_{l}(y)} & =\frac{g_{l}\left(y^{\prime}\right)}{g_{l}(y)} \cdot \frac{h\left(y^{\prime}\right)}{h(y)} \cdot \frac{h\left(\varphi^{l} y^{\prime}\right)}{h\left(\varphi^{l} y\right)} \\
& \leq \exp \left(C_{2} \Delta\right) \exp \left(2 C_{3} \Delta\right) \stackrel{\text { def }}{=} C .
\end{aligned}
$$

Therefore $C p_{l}(y) \geq \mu\left(B_{l}(y)\right) \geq C^{-1} p_{l}(y)$ and

$$
\int f_{+} d m \geq \frac{\gamma}{4} C^{-1} \cdot \sum_{y \in Q} p_{l}(y) \geq \frac{\gamma}{4} C^{-1} \frac{\gamma}{2}=\frac{\gamma^{2}}{8 C} .
$$


Since $m(f)=0$ then obviously

$$
\int \hat{f}_{-} d \mu=\int f_{-} d m=\int f_{+} d m \geq \frac{\gamma^{2}}{8 C} \stackrel{\text { def }}{=} \eta .
$$

Let us consider the set $S \subseteq M$ :

$$
S=\left\{z \in M: \hat{f}_{-}(z) \geq \eta / 2\right\} \text {. }
$$

Clearly, we have

$$
\mu(S) \cdot 1+\mu\left(\left\{f_{-} \leq \frac{\eta}{2}\right\}\right) \frac{\eta}{2} \geq \eta
$$

Hence

$$
\mu(S) \geq \eta / 2 .
$$

Let us consider the set

$$
Q_{1}=\left\{y \in \varphi^{-l}(x): B_{l}(y) \cap S \neq 0\right\} .
$$

By an argument similar to the one following estimate (47) we show that if $\delta_{1}=\varepsilon \eta / 8$ and $\Delta \lambda^{l} \geq \delta_{1}$, then

$$
\hat{f}\left(y^{\prime}\right) \leq-\eta / 4 \quad \text { as } y^{\prime} \in B_{l}(y) .
$$

Hence,

$$
\begin{aligned}
\sum_{y \in Q_{1}} \hat{f}(y) p_{l}(y) & \leq-\sum_{y \in Q_{1}} \frac{\eta}{4} \cdot C^{-1} \mu\left(B_{l}(y)\right) \\
& \leq-\frac{\eta}{4 C} \mu(S) \leq-\frac{\eta}{4 C} \cdot \frac{\eta}{2}=-\frac{\eta^{2}}{8 C} \\
& =-\frac{1}{8 C} \cdot\left(\frac{\gamma^{2}}{8 C}\right)^{2}=-\frac{\gamma^{4}}{(8 C)^{3}}
\end{aligned}
$$

Hence,

$$
\frac{P^{l} f(x)}{h(x)}=\sum_{y \in \varphi^{-l}(x) \backslash Q_{1}} \hat{f}(y) p_{l}(y)+\sum_{y \in Q_{1}} \hat{f}(y) p_{l}(y) \leq 1-\frac{\gamma^{4}}{(8 C)^{3}} .
$$

Because of the assumption $P^{l} f(x) / h(x) \geq \gamma$ we must have

$$
\gamma \leq 1-\frac{\gamma^{4}}{(8 C)^{3}} \text {. }
$$

This is false for $\gamma$ close to 1 . From now on we assume that $\gamma$ is set to the root of the equation $\gamma=1-\gamma^{4} /(8 C)^{3}$. In view of the above considerations we can write

$$
\left\|P^{l} f\right\| \leq \gamma\|f\|
$$


Also, in Case 2

$$
\begin{aligned}
\left\|d\left(P^{l} f\right)\right\| & \leq A \lambda^{l}\|d f\|+B\|f\| \\
& \leq \frac{A \lambda^{l}}{\varepsilon}+B \leq \frac{1}{R}\left(\frac{A \lambda^{l}}{\varepsilon}+B\right)\|\| f\|\| .
\end{aligned}
$$

We get

$$
\left|\left\|P ^ { l } f \left|\left\|\leq \max \left(\gamma, \frac{1}{R}\left(\frac{A \lambda^{l}}{\varepsilon}+B\right)\right)|\|f \mid\| .\right.\right.\right.\right.
$$

Now it is clear how we need to choose the constants. We ought to pick $\varepsilon, R$ and $l$ in such fashion that the numbers

$$
R \varepsilon, A \lambda^{l}+B \varepsilon, \gamma, \frac{1}{R}\left(A \frac{\lambda^{l}}{\varepsilon}+B\right)
$$

are all $<1$. Our choice is to make $R \varepsilon=\gamma$ and then

$$
\frac{1}{R}\left(A \frac{\lambda^{l}}{\varepsilon}+B\right)=\frac{A \lambda^{l}+B \varepsilon}{R \varepsilon} \leq \gamma .
$$

Or

$$
A \lambda^{l}+B \varepsilon \leq \gamma^{2}
$$

Since we required that $\lambda^{l} \leq \delta_{1} / \Delta \leq \varepsilon \gamma^{2} / 64 C \Delta$, it is sufficient to assume that

$$
\frac{A \varepsilon \gamma^{2}}{64 C \Delta}+B \varepsilon \leq \gamma^{2}
$$

or

$$
\frac{\varepsilon}{A \gamma^{2} / 64 C \Delta+B} \leq \gamma^{2}
$$

So, $\varepsilon, l$ should be picked to satisfy

$$
\lambda^{l} \leq \frac{\gamma^{4}}{A \gamma^{2}+64 B C \Delta} .
$$

A slightly more tractable choice of constants is possible by noticing that $\gamma \geq$ $1-\frac{4}{8^{3}}=\frac{127}{128}$ (always). Therefore it is easy to see that $\gamma \leq \exp \left(-1 /(9 C)^{3}\right)$. We notice that $\gamma^{4} \geq \frac{31}{32}$. We define a new constant:

$$
D=\frac{32}{31}(A+64 B C \Delta) \text {. }
$$

Subsequently we choose $l$ to be the smallest integer satisfying the inequality

$$
l \geq \frac{\log _{+}(D)}{\log (1 / \lambda)}
$$

It is easy to see that by making this choice we satisfy all previous requirements. We obtain

$$
\left\|P^{l}\right\|^{1 / l} \leq \gamma^{1 / l} \leq \exp \left(-1 /(9 C)^{3} l\right) .
$$

This concludes the proof. 
Corollary 2. Let $\rho(P \mid X)$ be the spectral radius of $P$ on $X$. Then

$$
\log \rho(P \mid X) \leq 1 /(9 C)^{3} l
$$

This estimate is the estimate of the mixing rate for $C^{1}$-functions on $M$.

\section{TWO CONSEQUENCES OF THEOREM 1}

In this section we describe in detail the two results based on Theorem 1 noted in the Introduction.

\subsection{Continuity of the invariant density.}

Theorem 2. Let $\mathscr{E}^{2} \subset C^{2}(M, M)$ be the open set of all expanding maps. Let $h_{\varphi} \in C^{1}\left(M, \mathbf{R}_{+}\right)$denote the density of the invariant measure of $\varphi \in \mathscr{E}^{2}$. Then:

1. The map $\mathscr{E}^{2} \ni \varphi \mapsto h_{\varphi} \in C^{0}(M, \mathbf{R})$ is continuous and has modulus of continuity $x \log (1 / x)$;

2. The map $\mathscr{E}^{2} \ni \varphi \mapsto h_{\varphi} \in C^{2}(M, \mathbf{R})$ is continuous (the modulus of continuity of this map depends on the modulus of continuity of the second partials of $\varphi$ in some local representation).

Proof. We need to show first that the map

$$
\mathscr{E}^{2} \ni \varphi \mapsto P_{\varphi} \in L\left(C^{1}(M, \mathbf{R}), C^{0}(M, \mathbf{R})\right)
$$

is Lipschitz (note the loss of differentiability).

Indeed, locally (i.e. using local coordinate systems) $P_{\varphi}$ can be written in the following form:

$$
P_{\varphi} f(x)=\sum_{j=1}^{N}\left(g_{\varphi} \cdot f\right) \circ \phi_{j}
$$

where $N=\operatorname{deg}(\varphi)$ and $g_{\varphi}$ is $1 / J_{\varphi}$, and $J_{\varphi}$ is the Jacobian of $\varphi$. The maps $\phi_{j}$ satisfy locally $\varphi \circ \phi_{j}=\mathrm{id}$. Suppose now that $\varphi=\varphi_{\alpha}$, where $\alpha$ is a parameter. Then

$$
\begin{aligned}
\frac{\partial}{\partial \alpha} P_{\varphi_{\alpha}} f(x)= & \sum_{j}\left(\frac{\partial g_{\varphi_{\alpha}}}{\partial \alpha} \cdot f\right) \circ \phi_{j} \\
& +\sum_{j} d\left(g_{\varphi_{\alpha}} \cdot f\right) \circ \phi_{j} \cdot \frac{\partial \phi_{j}^{\alpha}}{\partial \alpha} .
\end{aligned}
$$

Here $\phi_{j}^{\alpha} \circ \varphi_{\alpha}=$ id and $\varphi_{\alpha} \circ \phi_{j}^{\alpha}=\mathrm{id}$. The Implicit Function Theorem yields

$$
\frac{\partial \phi_{j}^{\alpha}}{\partial \alpha}=-\left[\left(D \varphi_{\alpha}\right)^{-1} \cdot \frac{\partial \varphi_{\alpha}}{\partial \alpha}\right] \circ \phi_{j}^{\alpha}
$$


We find easily that

$$
\begin{aligned}
\left|\frac{\partial}{\partial \alpha} P_{\varphi_{\alpha}} f\right| \leq & \frac{1}{g_{\varphi_{\alpha}}}\left|\frac{\partial}{\partial \alpha} g_{\varphi_{\alpha}}\right| P_{\varphi_{\alpha}}(|f|) \\
& +\sup \left(\frac{\mid d g_{\varphi_{\alpha}}}{g_{\varphi_{\alpha}}}\right) \cdot \sup \left|\frac{\partial \phi_{j}^{\alpha}}{\partial \alpha}\right| P_{\varphi_{\alpha}}|f| \\
& +\sup _{j}\left|\frac{\partial \phi_{j}^{\alpha}}{\partial \alpha}\right| P_{\varphi_{\alpha}}(|d f|) .
\end{aligned}
$$

This implies an estimate

$$
\left|\frac{\partial}{\partial \alpha} P_{\varphi_{\alpha}} f\right| \leq \text { const } \cdot\left\|\frac{\partial}{\partial \alpha} \varphi_{\alpha}\right\|_{C^{1}}\|f\|_{C^{1}} .
$$

This implies easily that if $\varphi, \psi \in \mathscr{E}^{2}$ then

$$
\left\|P_{\varphi}-P_{\psi}\right\| \leq \text { const } d(\varphi, \psi),
$$

where $d(\varphi, \psi)$ is the distance in $C^{1}$-topology and the constant depends on the $C^{1}$-norms of $T \varphi$ and $T \psi$ (tangent maps of $\varphi$ and $\psi$ ). Now we can discuss the solution to the equation

$$
\left(I-P_{\varphi}\right) f=P_{\varphi} h_{\psi}-h_{\psi}=u .
$$

Clearly, we have $\|u\|_{C^{1}} \leq$ const and in view of the equality $u=\left(P_{\varphi}-P_{\psi}\right) h_{\psi}$ and inequality (82) we also have $\|u\|_{C^{0}} \leq \operatorname{const} d(\varphi, \psi)$. Also $m(u)=0$. From Theorem 1 it follows that

$$
f=\left(I+P_{\varphi}+P_{\varphi}^{2}+\cdots\right) u
$$

exists and for any $N \in \mathbf{Z}_{+}$:

$$
\begin{aligned}
\|f\|_{C^{0}} & \leq\left\|\sum_{i=0}^{N-1} P_{\varphi}^{i} u+\sum_{i=N}^{\infty} P_{\varphi}^{i} u\right\| \\
& \leq \text { const } N d(\varphi, \psi)+\text { const } \cdot q^{N},
\end{aligned}
$$

where the constant $q \in(0,1)$ is given by Theorem 1 . The best estimate is obtained by taking $N \sim \log 1 / d(\varphi, \psi)$. This yields

$$
\|f\|_{C^{0}} \leq \operatorname{const} d(\varphi, \psi) \log \frac{1}{d(\varphi, \psi)} \text {. }
$$

This concludes the proof of the first claim of our theorem. To prove the second claim it suffices to show that $\|u\|_{C^{1}}$ goes to 0 , as $\varphi \rightarrow \psi$ in $C^{2}$-topology. Working again in local coordinates and differentiating formula (77) we obtain (for any $\left.f \in C^{1}(M, \mathbf{R})\right)$ :

$$
\begin{aligned}
d\left(P_{\varphi} f\right) & =\sum_{j=1}^{N} d\left(g_{\varphi} f\right) \circ \phi_{j} D \phi_{j} \\
& =\sum_{j=1}^{N}\left(f d g_{\varphi}\right) \circ \phi_{j} D \phi_{j}+\sum_{j=1}^{N}\left(g_{\varphi} d f\right) \circ \phi_{j} D \phi_{j}
\end{aligned}
$$


From this formula it is clear that the map $\mathscr{E}^{2} \ni \varphi \mapsto P_{\varphi} f \in C^{1}(M, \mathbf{R})$ is continuous. We complete our proof by applying this observation to $u=P_{\varphi} h_{\psi}-$ $P_{\psi} h_{\psi}$.

Remark 3. From the proof it follows that the map $\varphi \mapsto h_{\varphi}$ is continuous in $C^{1}$-topology on $\mathscr{E}^{2}$, provided that $T \varphi$ stays uniformly bounded in Lipschitz topology. The modulus of continuity is of the type $x \log (1 / x)$.

Corollary 3. The entropy $h_{\mu_{\varphi}}(\varphi)$ has modulus of continuity $x \log (1 / x)$.

4.2 An estimate of the rate of mixing. Based on the considerations outlined in the Introduction, the following theorem can be proved:

Theorem 3. If $f \in L^{1}(m)$ and $g \in C^{1}(M)$ then the sequence

$$
k_{n}=\int_{M} f \cdot g \circ \varphi^{n} d \mu-\int_{M} f d \mu \int_{M} f d \mu
$$

satisfies an inequality

$$
\left|k_{n}\right| \leq K\|f\|_{L^{1}}\|g\|_{C^{1}} r^{n},
$$

where $r \in(0,1)$ and $K>0$ can be chosen to depend on $C_{0}, C_{1}, \lambda$ and $\operatorname{diam}(M)$ only. Therefore, $K$ and $r$ are uniformly bounded for $\varphi$ in any compact subset of $\mathscr{E}^{2}$.

\section{REFERENCES}

1. M. Benedicks and L. Carleson, On iterations of $1-a x^{2}$ on $(-1,1)$, Ann. of Math. (2) 122 (1985), 1-25.

2. R. Bowen, Equilibrium states and the ergodic theory of Anosov diffeomorphisms, Lecture Notes in Math., vol. 470, Springer-Verlag, Berlin, Heidelberg and New York, 1975.

3. F. Hofbauer and G. Keller, Ergodic properties of invariant measures for piecewise monotonic transformations, Math. Z. 180 (1982), 119-140.

4. M. V. Jakobson, Absolutely continuous invariant measures for one-parameter families of onedimensional maps, Comm. Math. Phys. 81 (1981), 39-88.

5. K. Krzyżewski and W. Szlenk, On invariant measures for expanding differentiable mappings, Studia Math. 23 (1969), 83-92.

6. K. Krzyżesksi, On connection between expanding mappings and Markov chains, Bull. Acad. Polon. Sci. Ser. Math. 19 (1971), 291-293.

7. M. Rychlik, Bounded variation and invariant measures, Studia Math. 76 (1983), 69-80.

8. _ Another proof of Jakobson's theorem and related results, Ergodic Theory Dynamical Systems 8 (1988), 93-109.

9. M. Rychlik and E. Sorets, Additional properties of absolutely continuous invariant measures for the quadratic family, IAS, 1988 (in preparation).

10. Ya. G. Sinai, Gibbs measures in ergodic theory, Uspekhi Math. Nauk 27 (1972), 21-64.

11. P. Walters, Invariant measures and equilibrium states for some mappings which expand distances, Trans. Amer. Math. Soc. 236 (1978), 121-153.

12. Y. Yomdin, Volume growth and entropy, Israel J. Math. 57 (1987), 285-300. 08540

School of Mathematics, The Institute for Advanced Study, Princeton, New Jersey 